\title{
The 7th/8th American Joint Committee on Cancer and the Modified Union for International Cancer Control Staging System for Hepatocellular Carcinoma
}

\author{
In-Gyu Kim, Xu-Guang Hu, Hee-Jung Wang, Bong-Wan Kim, Sung Yeon Hong, and Xue-Yin Shen \\ Division of Liver Transplantation and Hepatobiliary Surgery, Department of Surgery, Ajou University School of Medicine, Suwon, Korea.
}

\begin{abstract}
Purpose: Although many staging systems have been proposed for hepatocellular carcinoma (HCC), there is no globally accepted system due to the extreme heterogeneity of the disease. We aimed to compare the results of the 7th/8th American Joint Committee on Cancer (AJCC) and the modified Union for International Cancer Control (mUICC) staging systems in patients with HCC. Materials and Methods: We collected data from 792 patients who underwent hepatic resection at our center. The Kaplan-Meier method was used to determine disease-free survival and overall survival. To evaluate homogeneity, '-2 log likelihood' was calculated using Cox proportional hazards regression. To measure discriminatory ability, the linear trend chi method and the CochranArmitage test for trend were used. The ability to accurately predict survival was verified by cross-validation analysis.

Results: Kaplan-Meier curves for disease-free survival and overall survival showed mUICC to be superior to the 7th/8th AJCC. The homogeneity test indicated that mUICC was the best for both disease-free survival and overall survival. In the discriminatory ability test, the chi-square value of mUICC was the best for disease-free survival, while the 7th AJCC had the best value for overall survival. In the cross-validation analysis, all three staging systems had significant predictive power.

Conclusion: mUICC seemed to be superior to the 7th/8th AJCC after analyzing the data of our surgical patients, although the geographic heterogeneity of HCC might result in differences between the staging systems. We believe that, while the three staging systems allow for the clear stratification of patients into prognostic groups, mUICC may be more appropriate in HCC.
\end{abstract}

Key Words: Hepatocellular carcinoma, staging, survival analysis

\section{INTRODUCTION}

Over the past 20 years, considerable progress has been made in the diagnosis and treatment of hepatocellular carcinoma (HCC). Hepatic resection, liver transplantation, radiofrequency ablation, and transcatheter arterial chemoembolization (TACE)

Received: July 9, 2018 Revised: November 10, 2018

Accepted: December 11, 2018

Corresponding author: Hee-Jung Wang, MD, PhD, Division of Liver Transplantation and Hepatobiliary Surgery, Department of Surgery, Ajou University School of Medicine, 164 World cup-ro, Yeongtong-gu, Suwon 16499, Korea.

Tel: 82-31-219-5200, Fax: 82-31-219-5755, E-mail: wanghj@ajou.ac.kr

Presented in part to Annual Congress of Korean Surgical Society 2016, Seoul, Korea (Nov 2016).

-The authors have no potential conflicts of interest to disclose.

(C) Copyright: Yonsei University College of Medicine 2019

This is an Open Access article distributed under the terms of the Creative Commons Attribution Non-Commercial License (https://creativecommons.org/licenses/ by-nc/4.0) which permits unrestricted non-commercial use, distribution, and reproduction in any medium, provided the original work is properly cited. have all been used to treat HCC according to clinicopathologic characteristics and hepatic function reserve. Notwithstanding, the optimal management for these patients remains controversial.

Staging systems subdivide patients with cancer into cohorts based on the extent and severity of the disease and predict survival at each level of severity. ${ }^{1}$ This subdivision enables assignment of a prognosis to the cohort that matches the staging criteria, and it allows clinicians to select the primary and adjuvant therapy. Although many staging and scoring systems have been proposed, there is currently no globally accepted system for assessing HCC due to the extreme heterogeneity of the disease. ${ }^{1,2}$ The prognosis and treatment of HCC depend on the patient's underlying liver disease and liver function reserve, in addition to the tumor biology. There are many useful staging systems, such as those of the American Joint Committee on Cancer (AJCC), Okuda, Barcelona Clinic Liver Cancer (BCLC), Cancer of Liver Italian Program (CLIP), modified Union of Interna- 
tional Cancer Control (mUICC), Chinese University Prognostic Index (CUPI), and French. ${ }^{3,4}$ The Korean Liver Cancer Group has recently adopted the mUICC as the main staging classification system for HCC, ${ }^{4,5}$ and they insist that the BCLC and the AJCC systems should also be considered as additional inclusions. Our institute has used the AJCC, the mUICC, and the BCLC staging systems for HCC. In this study, we compared the AJCC and the mUICC systems, assessing data on patients who underwent surgical resection. Here, we present the results of the AJCC (7th and 8th) and the mUICC staging systems applied to patients with HCC, comparing their homogeneity, discriminatory ability, and predictive power for 792 patients who underwent hepatic resection at Ajou University Medical Center in South Korea.

\section{MATERIALS AND METHODS}

This study was performed in accordance with the ethical guidelines of the 1975 Declaration of Helsinki and was approved by Ajou University Hospital Institutional Review Board (approval code: AJIRB-MED-MDB-17-204). Between April 1994 and December 2013, 792 consecutive patients were included, and they were followed until June 2017. All patients included in this study underwent surgical resection (liver transplantation excluded). The variables for the demographics of the HCC cohort were sex, age, hepatitis B surface antigen (HBsAg), hepatitis C antibody (Anti-HCV), serum total bilirubin, serum albumin, ICG-R15 (indocyanine green retention rate at 15 minutes), prothrombin time, and alpha-fetoprotein. We added calculated hepatic venous pressure gradient (cHVPG) as a preoperative variable for reserve function of the liver, which we developed recently. ${ }^{6}$ Peri-operative characteristics included major/minor resection, operative time, bleeding and transfusion during surgery, post-operative complication, in-hospital mortality (within 1 month after surgery), and length of stay after surgery. The pathologic factors were tumor size, number of tumor, portal vein invasion, hepatic vein invasion, bile duct invasion, histologic grade, degree of fibrosis of background liver, and macroscopic intrahepatic metastasis. Tumor size was based on the largest dimension of the tumor specimen. The number of HCCs was defined by the total number of nodules, including intrahepatic metastasis, in the resected specimen. Microvascular invasion and minor vascular invasion were considered vascular invasion of T2 in the 7th and 8th AJCC, and invasion of a major branch (1st order) to a portal vein/ hepatic vein/ hepatic artery was considered major vascular invasion of T3b in the 7th and T4 in the 8th. For the mUICC, major and minor vascular invasion of portal vein, hepatic vein, and bile duct were considered in staging, and microvascular invasion was excluded in staging.

Statistical analyses were performed with the Statistical Package for the Social Sciences (SPSS) version 23.0 (IBM Corp., Ar- monk, NY, USA) for most of the analyses. All deaths were counted as events, and living patients were censored to the date of the last follow-up. Disease-free survival was measured from the time of hepatic resection, and recurrence was the endpoint. Overall survival was measured from the time of hepatic resection, and death was the endpoint. Survival curves were constructed using the Kaplan-Meier method and compared using a log-rank test with the Bonferroni correction. In order to identify significant prognostic factors of HCC, univariable and multivariable Cox's regression analyses were performed. The overall survival and disease-free survival models were tested for appropriateness of proportional hazard assumption.

To evaluate homogeneity (smaller differences in survival among patients of the same stage) within each staging system, '-2 log likelihood' was calculated using Cox proportional hazards regression. ${ }^{7}$ To measure the discriminatory ability (greater differences in survival between each of the stages) of each staging system, the linear trend chi method was used. ${ }^{7}$ We also applied the Cochran-Armitage test for trend to evaluate linear trends; this was performed with the Statistical Analysis System (SAS), version 9.4 (SAS Institute Inc., Cary, NC, USA).

The ability of the AJCC classification and the mUICC classification to accurately predict survival was verified and compared by cross-validation analysis. ${ }^{8,9}$ Patients were randomly divided into a test sample group and a validation sample group. We obtained Pearson's correlation coefficient ' $r$ ' in the test sample and validation sample, respectively. We compared the predictive accuracy in terms of ' $z$ ' between the three staging systems, using the Fisher r-to-z transformation method.

We also used SAS for cross-validation analysis. Although this was a similar method to the cross-validation analysis using SPSS, we applied it using SAS for further confirmation of the predictive accuracy. Statistical significance was defined as a $p$ value $<0.05$.

\section{Selection of surgical resection for patients with HCC} Child-Turcotte-Pugh (CTP) classification is conventionally used to pre-operatively assess the safety of hepatic resection. In general, only as a class of A or B is an indication for surgical resection. An Eastern Cooperative Oncology Group (ECOG) performance status 0-2 and American Society of Anesthesiologists (ASA) class 1-3 are also generally accepted indications. For assessment of reservoir function after surgery, we used ICGR15 and Y-value to decide resection volume after measuring liver volume. ${ }^{10,11}$ A remnant liver volume of $40 \%$ or more is generally recommended in cirrhotic patients for safety. ${ }^{12}$ We recommend surgery in cases of less than 20\% of ICG-R15 and a Y-value less than 45 . We have recently added CHVPG, and we recommend surgery in cases of where cHVPG is less than 10 $\mathrm{mm} \mathrm{Hg} .{ }^{6}$ For preoperative staging in our multidisciplinary team meeting, we use BCLC, Okuda stage, AJCC, and mUICC. In the case of surgery, we created a follow-up plan for the patient and collected data prospectively using the AJCC and 
mUICC staging systems. Curative resection was defined as that in which the entire tumor could be removed macroscopically. Lymph node involvement and distant metastasis were based on macroscopic inspection and palpation at the time of surgery, in addition to imaging study pre-operatively. We did not routinely perform lymph node dissection during hepatic resection for HCC unless preoperative imaging showed lymph node metastasis or we found lymph node enlargement in the operative field. For stage IVA of the AJCC and one type of stage IVA (anyTN1M0) of the mUICC, we aimed to perform surgery with curative intention according to reports from other authors, ${ }^{13-15}$ and we also actively performed follow-up for treatment after common recurrence. For stage IVB, we rarely recommended surgery after a multidisciplinary team discussion of each case. ${ }^{16}$

\section{RESULTS}

We have included many tables throughout this study, and we have placed many of them into the supplementary information. We believe these tables are necessary for fully understanding our data related to staging systems, allowing the readers to follow our paper more easily.

The pre- and peri-operative characteristics of the patients are shown in Supplementary Table 1 (only online). The median age was 53 years, and 609 patients $(76.9 \%)$ were male. HBsAg was positive in 599 cases (75.6\%), and anti-HCV was positive in 45 cases $(5.7 \%)$. The median platelet count was $153000 / \mu \mathrm{L}$, and the median serum total bilirubin was $0.7 \mathrm{mg} / \mathrm{dL}$. The serum aspartate aminotransferase (AST) and alanine aminotransferase (ALT) levels were $41 \mathrm{U} / \mathrm{L}$ and $39 \mathrm{U} / \mathrm{L}$ on average, respectively. The median serum albumin was $4.0 \mathrm{~g} / \mathrm{dL}$, and the median international normalized ratio of prothrombin time was 1.06. The median alpha-fetoprotein was $43.7 \mathrm{ng} / \mathrm{mL}$. CTP class A comprised 742 cases (93.7\%), with class B totaling 36 cases $(4.5 \%)$ and class C 14 cases (1.8\%). ICG-R15 was $13.5 \%$ on average, and cHVPG was $5.1 \mathrm{~mm} \mathrm{Hg}$ on average. Pre-operative TACE was done in 252 cases (31.8\%). Major resection was undertaken in 308 cases (38.9\%), which was defined as resection of the liver more than two Couinaud's segments. Operative time was 195 minutes on average. Bleeding during surgery was $700 \mathrm{~mL}$ on average. The median size of tumor was $4.0 \mathrm{~cm}$, and tumor number was 1.0 on average. Post-operative complication happened in 80 cases $(10.1 \%)$. The median length of hospital stay after surgery was 14 days. In-hospital mortality was recorded in 8 cases (1.0\%).

The distribution of patients and survival rate according to the three staging systems are shown in Supplementary Table 2 (only online). The total recurrence was 452 out of 792 (57.1\%) cases during the follow-up period. For the 7th AJCC, 325 of 792 (41.0\%) cases were stage I, 307 (38.8\%) were stage II, 51 (6.4\%) were stage IIIA, 45 (5.7\%) were stage IIIB, and 58 (7.3\%) were stage
IIIC. For the mUICC, 101 of $792(12.8 \%)$ cases were stage I, 400 (50.5\%) were stage II, 232 (29.3\%) were stage III, and $53(6.7 \%)$ were stage IVA (T4N0M0). For the 8th AJCC, 109 of 792 (13.8\%) cases were stage IA, 235 (29.7\%) were stage IB, 288 (36.4\%) were stage II, 51 (6.4\%) were stage IIIA, and 103 (13.0\%) were stage IIIB. The number of cases with an advanced stage, such as stage IVA and stage IVB, was very low for all three staging systems because we only enrolled patients who underwent surgical treatment. Based on the 7th AJCC, the 5-year disease-free survival rate of stage I was $61.5 \%$, stage II was $36.8 \%$, stage IIIA was $17.7 \%$, stage IIIB was $14.8 \%$, and stage IIIC was $18.7 \%$. For 5 -year overall survival rate, stage I was $86.8 \%$, stage II was $63.5 \%$, stage IIIA was $38.2 \%$, stage IIIB was $28.9 \%$, and stage IIIC was 28.6\%. Based on mUICC, 5-year disease-free survival rate of stage I was $60.8 \%$, stage II was $49.0 \%$, stage III was $35.5 \%$, and stage IVA (T4N0M0) was $6.8 \%$. For 5-year overall survival rate, stage I was $90.7 \%$, stage II was $75.6 \%$, stage III was $52.6 \%$, and stage IVA (T4N0M0) was 18.1\%. Based on the 8th AJCC, 5-year disease-survival rate of stage IA was $63.9 \%$, stage IB was $60.0 \%$, stage II was $35.3 \%$, stage IIIA was $17.7 \%$, and stage IIIB was $19.8 \%$. For 5-year overall survival rate, stage IA was $90.4 \%$, stage IB was $85.0 \%$, stage II was $62.0 \%$, stage IIIA was $38.2 \%$, and stage IIIB was $29.1 \%$.

Univariable and multivariable analyses in relation to diseasefree survival and overall survival for HCC patients are shown in Supplementary Tables 3 and 4 (only online). Multivariable analyses of the disease-free survival and overall survival models were tested for appropriateness of the proportional hazard assumption. The test based on Schoenfeld residuals showed that the variables met the proportional hazard assumption (Global $p=0.143$ for disease-free survival and $p=0.468$ for overall survival) when we excluded variables, like 'intrahepatic metastasis (IM)' that did not meet the proportional hazard assumption. For disease-free survival (Supplementary Table 3, only online), multivariable Cox regression showed hepatic vein invasion had the greatest impact, followed by serum albumin level $(<3 \mathrm{~g} / \mathrm{dL})$, tumor size $(>2 \mathrm{~cm})$, and positive margin. For overall survival (Supplementary Table 4, only online), multivariable Cox regression showed CTP class C and B had the greatest significance, followed by tumor number (multiple), microvascular invasion, and positive margin.

Six cases of stage IVA and IVB (one type of IVA in mUICC, anyTN1M0) were excluded in the Kaplan-Meier curve. A similar percentage and patterns of censored cases were shown in each stage of 7th AJCC, 8th AJCC, and mUICC. Therefore, we believe the assumption that censoring is reasonably similar in all groups was fulfilled. Fig. 1 shows the Kaplan-Meier curve with disease-free survival and overall survival of patients with 95\% confidence interval according to the 7th AJCC staging system. For disease-free survival, there were no significant differences among stage IIIA, stage IIIB, and stage IIIC, although there were significant differences among stage I, stage II, and stage IIIA. For overall survival, there was a significant difference 
between stage I and stage II, but there were no significant differences among other stages. Fig. 2 shows disease-free survival and overall survival of the 8th AJCC staging system. For diseasefree survival, there were significant differences among stage IB, stage II, and stage IIIA. For overall survival, there were signifi-

A

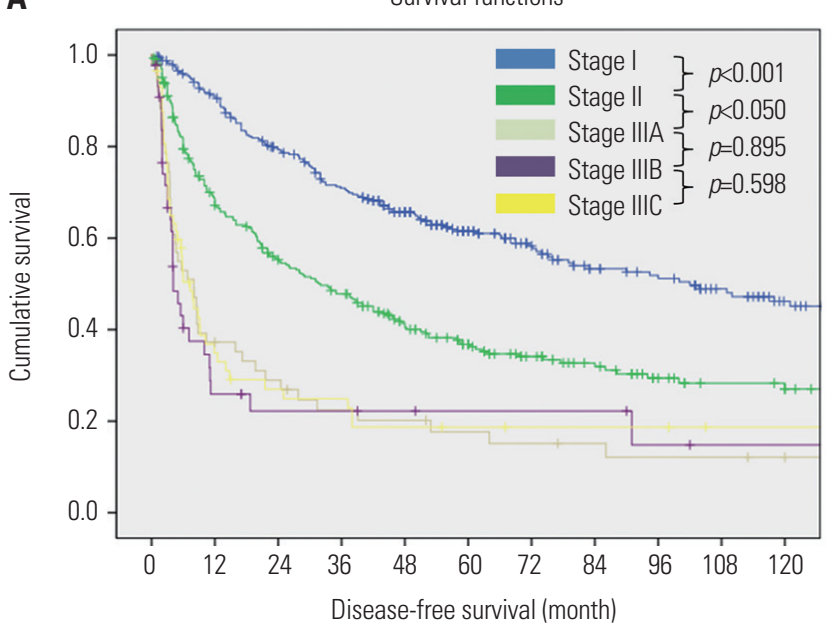

No. at risk

$\begin{array}{lrrrrrrrrrrr}\text { Stage I } & 325 & 285 & 240 & 210 & 173 & 127 & 97 & 78 & 71 & 57 & 43 \\ \text { Stage II } & 307 & 192 & 149 & 127 & 93 & 73 & 54 & 41 & 29 & 23 & 21 \\ \text { Stage IIIA } & 51 & 18 & 14 & 10 & 9 & 7 & 6 & 5 & 4 & 4 & 2 \\ \text { Stage IIIB } & 45 & 9 & 6 & 6 & 5 & 4 & 4 & 4 & 2 & 1 & 1 \\ \text { Stage IIIC } & 58 & 18 & 13 & 12 & 9 & 8 & 7 & 7 & 7 & 5 & 5\end{array}$

cant differences among stage IB, stage II, and stage IIIA. Fig. 3 shows disease-free survival and overall survival of the mUICC staging system. For disease-free survival, there were significant differences among stage II, stage III, and stage IVA (T4N0M0). For overall survival, all stages had significant differences among

B

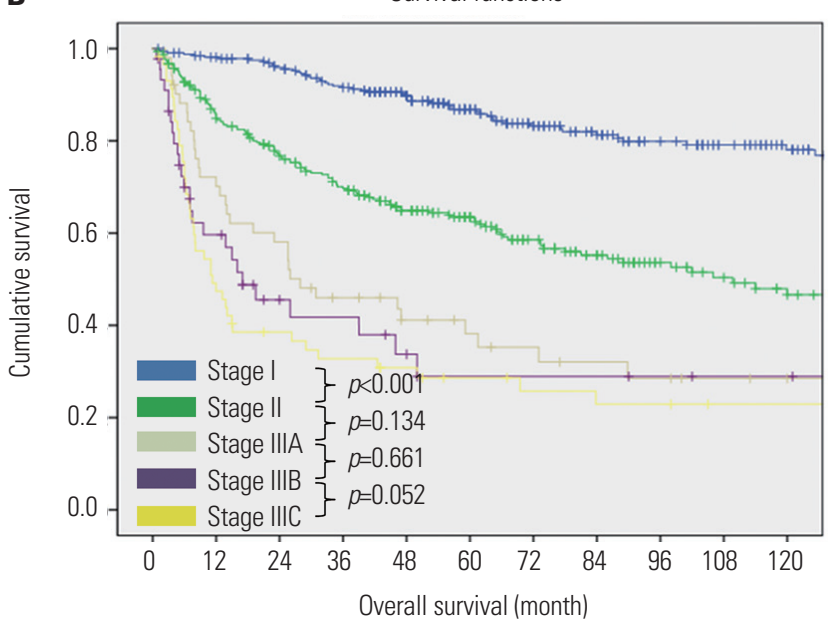

No. at risk

$\begin{array}{lrrrrrrrrrrr}\text { Stage I } & 325 & 311 & 294 & 274 & 232 & 178 & 143 & 122 & 105 & 89 & 74 \\ \text { Stage II } & 307 & 248 & 211 & 185 & 149 & 126 & 93 & 72 & 52 & 43 & 35 \\ \text { Stage IIIA } & 51 & 35 & 29 & 21 & 16 & 13 & 11 & 9 & 8 & 6 & 4 \\ \text { Stage IIIB } & 45 & 23 & 12 & 11 & 7 & 5 & 5 & 5 & 4 & 3 & 3 \\ \text { Stage IIIC } & 58 & 27 & 20 & 17 & 14 & 11 & 9 & 8 & 8 & 6 & 6\end{array}$

Fig. 1. Disease-free survival and overall survival in 7th American Joint Committee on Cancer (AJCC). (A) Kaplan-Meier curve shows disease-free survival of patients with $95 \%$ confidence interval according to 7 th AJCC staging system. $p$ values were from log-rank test. (B) Kaplan-Meier curve shows overall survival of patients with $95 \%$ confidence interval according to 7 th AJCC staging system. $p$ values were from log-rank test.

A

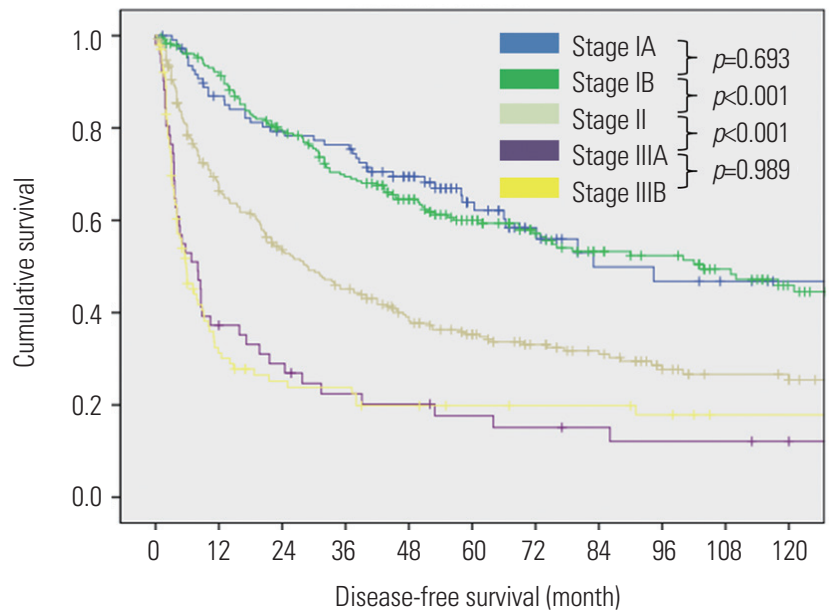

No. at risk

$\begin{array}{lrrrrrrrrrrr}\text { Stage IA } & 109 & 91 & 82 & 79 & 59 & 37 & 24 & 16 & 15 & 13 & 10 \\ \text { Stage IB } & 235 & 209 & 173 & 146 & 121 & 95 & 74 & 61 & 56 & 44 & 33 \\ \text { Stage II } & 288 & 177 & 134 & 111 & 85 & 68 & 53 & 42 & 30 & 23 & 21 \\ \text { Stage IIIA } & 51 & 18 & 14 & 10 & 9 & 7 & 6 & 5 & 4 & 4 & 2 \\ \text { Stage IIIB } & 103 & 27 & 19 & 18 & 14 & 12 & 11 & 11 & 9 & 6 & 6\end{array}$

B

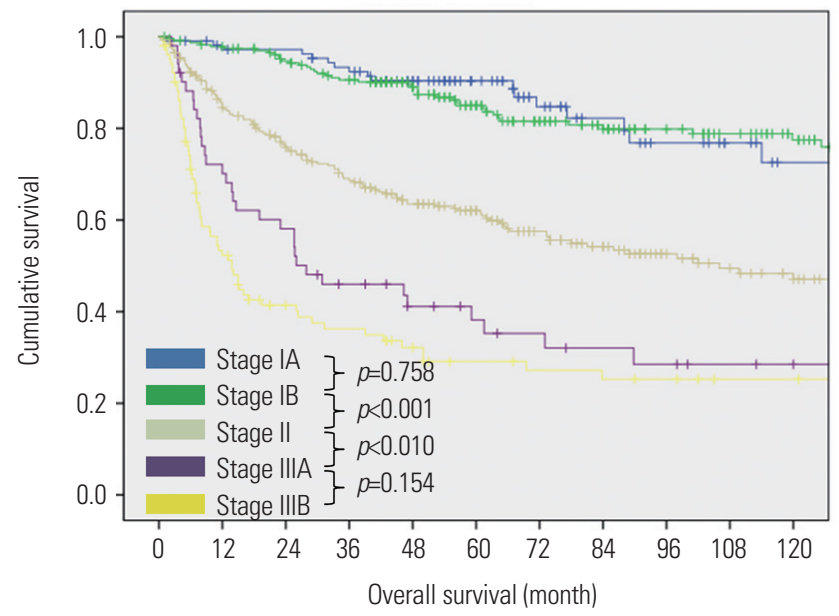

No. at risk

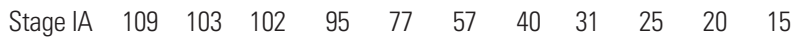
$\begin{array}{llllllllllll}\text { Stage IB } & 235 & 225 & 209 & 194 & 164 & 130 & 106 & 91 & 79 & 68 & 57\end{array}$ $\begin{array}{llllllllllll}\text { Stage II } & 288 & 231 & 194 & 169 & 139 & 117 & 90 & 72 & 53 & 44 & 37\end{array}$ $\begin{array}{llllllllllll}\text { Stage IIIA } & 51 & 35 & 29 & 21 & 16 & 13 & 11 & 9 & 8 & 6 & 4\end{array}$

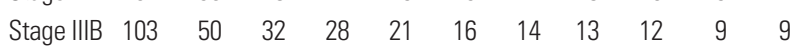

Fig. 2. Disease-free survival and overall survival in 8th American Joint Committee on Cancer (AJCC). (A) Kaplan-Meier curve shows disease-free survival of patients with $95 \%$ confidence interval according to 8th AJCC staging system. $p$ values were from log-rank test. (B) Kaplan-Meier curve shows overall survival of patients with $95 \%$ confidence interval according to 8th AJCC staging system. $p$ values were from log-rank test. 
A

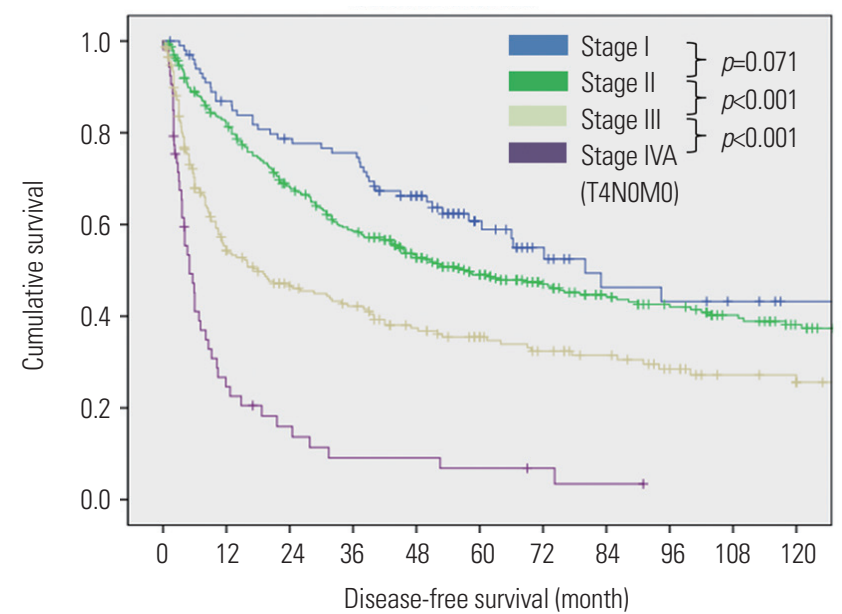

No. at risk

$\begin{array}{llllllllllll}\text { Stage I } & 101 & 85 & 76 & 73 & 55 & 33 & 22 & 15 & 14 & 12 & 9\end{array}$

$\begin{array}{llllllllllll}\text { Stage II } & 400 & 316 & 253 & 213 & 171 & 135 & 104 & 85 & 74 & 60 & 48\end{array}$

$\begin{array}{llllllllllll}\text { Stage III } & 232 & 111 & 86 & 75 & 59 & 48 & 40 & 34 & 25 & 18 & 16\end{array}$

Stage IVA

(T4NOMO)
B

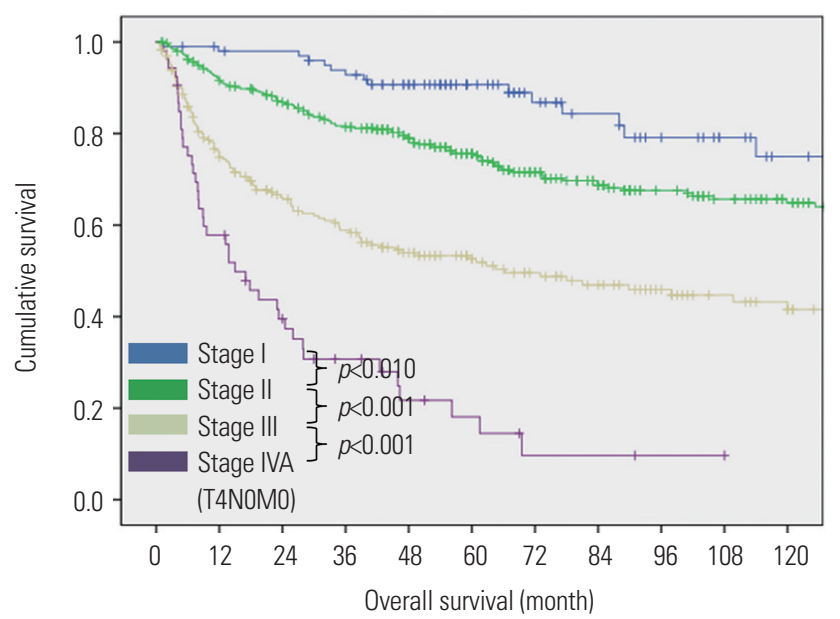

No. at risk

$\begin{array}{llllllllllll}\text { Stage I } & 101 & 97 & 96 & 89 & 75 & 55 & 41 & 33 & 26 & 21 & 16\end{array}$

$\begin{array}{llllllllllll}\text { Stage II } & 400 & 357 & 323 & 295 & 246 & 201 & 158 & 132 & 112 & 96 & 82\end{array}$

$\begin{array}{llllllllllll}\text { Stage III } & 232 & 162 & 128 & 112 & 89 & 72 & 60 & 49 & 38 & 30 & 25\end{array}$

Stage IVA

(T4NOMO)

Fig. 3. Disease-free survival and overall survival in modified Union for International Cancer Control (mUICC). (A) Kaplan-Meier curve shows disease-free survival of patients with $95 \%$ confidence interval according to mUICC staging system. $p$ values were from log-rank test. (B) Kaplan-Meier curve shows overall survival of patients with $95 \%$ confidence interval according to mUICC staging system. $p$ values were from log-rank test.

their stages. We then performed Bonferroni correction for logrank test (Supplementary Tables 5-10, only online). To summarize, Kaplan-Meier curves for disease-free survival and overall survival showed that the mUICC was superior to the 7th and 8th AJCC.

The homogeneity test results are shown in Table 1. Generally, more accurate stages showed lower ' -2 log likelihoods." For disease-free survival, mUICC was the best because the ' -2 log likelihood' had the lowest value. For overall survival, mUICC was also the best because of its lowest ' -2 log likelihood' among the three staging systems.

The discriminatory ability test is shown in Table 2. Generally, a higher linear trend chi-square value indicates better discriminatory ability. ${ }^{7}$ For disease-free survival, the Pearson chi-square value was the highest in the mUICC, followed by the 8th AJCC and 7th AJCC. For overall survival, the 7th AJCC had the highest Pearson chi-square value, followed by mUICC and the 8th AJCC. In Cochran-Armitage test for linear trend, the value of the Cochran-Armitage test was the highest in 8th AJCC, followed by mUICC and 7th AJCC, for disease-free survival. For overall survival, the mUICC had the highest value of CochranArmitage test, followed by the 8th AJCC and the 7th AJCC.

The results of cross-validation analysis using SPSS are shown in Table 3. For disease-free survival, all three stages had significant predictive power. The ' $z$ ' value in the Fisher r-to-z transformation between the test sample and validation sample was the lowest in 8th AJCC, followed by 7th AJCC and mUICC. For overall survival, all three stages had significant predictive power. The 7th AJCC had the lowest ' $z$ ' value, followed by
Table 1. Homogeneity Analysis of the Three Staging Systems

\begin{tabular}{cccccc}
\hline \multicolumn{2}{c}{ Disease-free survival } & & \multicolumn{2}{c}{ Overall survival } \\
\cline { 1 - 2 } \cline { 5 - 6 } $\begin{array}{c}\text { Staging } \\
\text { system }\end{array}$ & $\begin{array}{c}\mathbf{- 2 ~ l o g} \\
\text { likelihood }\end{array}$ & & $\begin{array}{c}\text { Staging } \\
\text { system }\end{array}$ & $\begin{array}{c}\mathbf{- 2} \text { log } \\
\text { likelihood }\end{array}$ \\
\hline 7th AJCC & $5427.820^{*}$ & & 7th AJCC & $3419.374^{*}$ \\
8th AJCC & $5423.002^{*}$ & & 8th AJCC & $3421.065^{*}$ \\
mUICC & $5413.979^{*}$ & & mUICC & $3417.959^{*}$ \\
\hline
\end{tabular}

AJCC, American Joint Committee on Cancer; mUICC, modified Union of International Cancer Control.

${ }^{*} p<0.001$

mUICC and 8th AJCC, although the ' $z$ ' value of 8th AJCC was much higher than the others.

The second trial of the cross-validation analysis using SAS is shown in Supplementary Tables 11-14 (only online).

For disease-free survival, the mUICC was better than the 7th AJCC, because $p$ values (for ' $\mathrm{Pr}>\mathrm{ChiSq}$ ' in Supplementary Table 11 , only online) of mUICC in both the test and validation samples had more significant ones. The mUICC was also better than the 8th AJCC based on the same reason.

For overall survival, the mUICC and the 7th AJCC had significant predictive power, and were comparable with each other in the test sample and validation sample. For mUICC and the 8th AJCC, we could not conclude which is better between mUICC and the 8th AJCC because the results of test sample and validation sample did not show consistent $p$ values. 
In-Gyu Kim, et al.

Table 2. Discriminatory Ability Analysis of the Three Staging Systems

\begin{tabular}{|c|c|c|c|c|c|c|c|}
\hline \multicolumn{4}{|c|}{ Disease-free survival } & \multicolumn{4}{|c|}{ Overall survival } \\
\hline $\begin{array}{l}\text { Staging } \\
\text { system }\end{array}$ & $\begin{array}{c}\text { Pearson } \\
\text { chi-square }\end{array}$ & Linear-by-Linear & $\begin{array}{l}\text { Cochran-Armitage } \\
\text { Test for trend }\end{array}$ & $\begin{array}{l}\text { Staging } \\
\text { system }\end{array}$ & $\begin{array}{c}\text { Pearson } \\
\text { chi-square }\end{array}$ & Linear-by-Linear & $\begin{array}{c}\text { Cochran-Armitage } \\
\text { Test for trend }\end{array}$ \\
\hline 7th AJCC & $26.974^{*}$ & $18.338^{*}$ & $15.827^{*}$ & 7th AJCC & $90.925^{*}$ & $71.265^{*}$ & $71.355^{*}$ \\
\hline 8th AJCC & $31.001^{*}$ & $23.658^{*}$ & $21.147^{*}$ & 8th AJCC & $86.846^{*}$ & $73.645^{*}$ & $73.738^{*}$ \\
\hline mUICC & $32.551^{*}$ & $23.355^{*}$ & $20.305^{*}$ & mUICC & $88.699 *$ & $73.869^{*}$ & $73.847^{*}$ \\
\hline
\end{tabular}

AJCC, American Joint Committee on Cancer; mUICC, modified Union of International Cancer Control.

${ }^{*} p<0.001$.

Table 3. Cross-Validation Analysis of the Three Staging Systems Using SPSS

\begin{tabular}{cccccccccc}
\hline \multicolumn{1}{c}{$\begin{array}{c}\text { Staging } \\
\text { system }\end{array}$} & Test sample & $\begin{array}{c}\text { Validation } \\
\text { sample }\end{array}$ & $\begin{array}{c}\text { Fisher } r \text {-to- } \\
\text { transformation }\end{array}$ & & $\begin{array}{c}\text { Staging } \\
\text { system }\end{array}$ & & Test sample & $\begin{array}{c}\text { Validation } \\
\text { sample }\end{array}$ & $\begin{array}{c}\text { Fisher r-to-z } \\
\text { transformation }\end{array}$ \\
\hline 7th AJCC & $r=0.251(n=357)$ & $r=0.223(n=435)$ & $z=0.41, p=0.682$ & & 7 th AJCC & & $r=0.278(n=387)$ & $r=0.276(n=405)$ & $z=0.03, p=0.976$ \\
8th AJCC & $r=0.237(n=387)$ & $r=0.279(n=405)$ & $z=0.04, p=0.968$ & & 8 th AJCC & & $r=0.336(n=363)$ & $r=0.218(n=429)$ & $z=1.79, p=0.074$ \\
mUICC & $r=0.279(n=385)$ & $r=0.219(n=407)$ & $z=0.9, p=0.368$ & & mUICC & & $r=0.305(n=383)$ & $r=0.258(n=409)$ & $z=0.72, p=0.472$ \\
\hline
\end{tabular}

SPSS, Statistical Package for the Social Sciences; AJCC, American Joint Committee on Cancer; mUICC, modified Union of International Cancer Control; $r$, Pearson correlation coefficient; $n$, number of sample; $z$, Fisher's value of r-to-z transformation.

\section{DISCUSSION}

Although the BCLC has long been the dominant system for treatment-guided staging of HCC, some other authors do not fully agree with its underlying principles. ${ }^{4,5}$ One of the weaknesses of the BCLC is that it was not developed from a cohort of HCC patients based on multivariable analysis; therefore, it is difficult to predict the mortality of HCC patients. ${ }^{4,17,18}$ BCLC is the only staging system that assigns treatment strategies based on specific prognostic subclasses stratified according to the patient's tumor characteristics, baseline liver function, and overall health status. ${ }^{19}$ Most physicians agree that these concepts are important, but some would maintain that BCLC increases the complexity of immediate clinical staging. ${ }^{1,19}$

Both the AJCC and the mUICC staging systems were developed based on survival analysis of patients who underwent hepatic resection. The AJCC developed the TNM staging system, and it has been updated regularly since the first edition was published in $1977 .^{20,21}$ Vauthey, et al. ${ }^{22}$ developed a simplified staging system for HCC, which was adopted as the TNM staging system of the AJCC after minor changes. Several reports have shown that the prognostic ability of the 7th TNM system is poorer than that of BCLC classification, particularly in patients with advanced-stage HCC. ${ }^{7,23,24}$ The mUICC staging system originated from the Liver Cancer Study Group of Japan (LCSGJ), and Minagawa, et al. ${ }^{8}$ reported evidence for the development of the TNM system, validated the system, and compared its discriminatory ability and predictive power to those of the AJCC staging system in 13772 patients who underwent curative hepatic resection. They insisted that mUICC staging may be more appropriate for stratifying patients with earlystage HCC.

There are two types of staging systems for HCC. One is clini- cal staging, such as BCLC, Japanese Integrated System score (JIS), and CLIP, which can be applied in both operable and nonoperable patients: these have been described as 'medical staging. $^{1,8}$ The other type of staging system is based on pathologic staging, such as AJCC and mUICC, which can be applied in patients eligible for surgery: these have been described as 'surgical staging., ${ }^{1,8}$ We think multiple staging systems complicate the daily practice and academic activities of liver surgeons, and they keep clinicians from carefully using the currently available staging systems or treatment algorithms and from becoming familiar with their features and limitations. ${ }^{3}$ For instance, Kudo, et al. ${ }^{25,26}$ developed the new staging of JIS score based on LCSGJ, and other authors sought to modify it with some significant prognostic variables. ${ }^{27-29}$ One of the reasons why they developed a new staging system was that the LCSGJ required 12 groups (three liver disease stages multiplied by four tumor stages), which could be complicated in clinical practice. We tried applying the JIS score to our patient cohort; however, we did not find a difference in the survival curves from the mUICC in this study (Supplementary Fig. 1, only online). This result would suggest that the mUICC is sufficient without adding other variables for staging HCC patients who have undergone surgical resection. One reason for the sufficiency of mUICC is that most patients who underwent surgery have relatively good liver function, compared with inoperable patients.

As a whole, mUICC seemed to be superior to AJCC in this study. First, we suggest it may be related to the management problem with our data. However, we consecutively and prospectively collected the data of the patients, and this is the same as extracting routinely collected data from existing sources, such as electronic health record or registries. ${ }^{30}$ This approach does not interfere with routine practice nor require additional data 
collection. Second, bias or systematic error may be present, which is found in almost all scientific papers. The term 'bias' describes the systematic tendency of any factors associated with the design, implementation, analysis and interpretation of the results of the studies to estimate how the treatment derivates from its true value. We did our best to reduce the bias as much as possible. The mUICC and the AJCC were applied to the same clinical data, and we introduced significantly more statistical methods compared with other prior articles for HCC staging systems. Third, 'by chance', bias could happen within $p$ values, called 'random error'. More studies of other datasets from multiple centers are needed to solve this problem. Last, the mUICC should be more powerful than AJCC from academic and scientific perspectives after analyzing the data of our center, although the geographic heterogeneity observed in HCC might result in differences between mUICC and AJCC. Vauthey, et al. ${ }^{22}$ proposed a simplification of the TNM staging system and identified independent prognostic factors. They recommended that the T-component focus on vascular invasion, tumor number, and tumor size, which corresponded almost completely with those that were introduced in the mUICC staging system as significant prognostic variables.

The results of this study support the use of the mUICC staging system over the AJCC staging system. While both staging systems allow for the clear stratification of patients into prognostic groups, we believe the mUICC staging system may be more appropriate for stratifying patients with HCC. In addition, we suggest that it may be sufficient to use a surgical/pathologic staging system, such as the mUICC or the AJCC, when surgical resection is performed in patients with HCC.

\section{ACKNOWLEDGEMENTS}

The authors thank J Han, PhD, working at Office of Biostatics, Ajou University School of Medicine for her excellent statistical assistance, especially in cross-validation using SAS.

\section{AUTHOR CONTRIBUTIONS}

Conceptualization: In-Gyu Kim, Xu-Guang Hu, Hee-Jung Wang. Data curation: In-Gyu Kim, Xu-Guang Hu, Sung Yeon Hong, Xue-Yin Shen. Formal analysis: In-Gyu Kim, Xu-Guang Hu, Bong-Wan Kim, Sung Yeon Hong, Xue-Yin Shen. Investigation: In-Gyu Kim, Xu-Guang Hu, Bong-Wan Kim, Sung Yeon Hong, Xue-Yin Shen. Methodology: InGyu Kim, Xu-Guang Hu, Bong-Wan Kim, Sung Yeon Hong, Xue-Yin Shen. Project administration: Hee-Jung Wang. Resources: Hee-Jung Wang, Bong-Wan Kim. Supervision: Hee-Jung Wang, Bong-Wan Kim. Validation: In-Gyu Kim. Visualization: In-Gyu Kim, Xu-Guang Hu. Writing-original draft: In-Gyu Kim, Hee-Jung Wang. Writing-review \& editing: In-Gyu Kim, Hee-Jung Wang.

\section{ORCID iDs}

In-Gyu Kim

Xu-Guang Hu https://orcid.org/0000-0001-8012-4334

https://orcid.org/0000-0002-2732-4916
Hee-Jung Wang

Bong-Wan Kim

Sung Yeon Hong

Xue-Yin Shen

https://orcid.org/0000-0002-0687-007X

https://orcid.org/0000-0001-9059-0451

https://orcid.org/0000-0001-6993-9583

https://orcid.org/0000-0001-6947-3509

\section{REFERENCES}

1. Henderson JM, Sherman M, Tavill A, Abecassis M, Chejfec G, Gramlich T. AHPBA/AJCC consensus conference on staging of hepatocellular carcinoma: consensus statement. HPB (Oxford) 2003; 5:243-50.

2. Subramaniam S, Kelley RK, Venook AP. A review of hepatocellular carcinoma (HCC) staging systems. Chin Clin Oncol 2013;2:33.

3. Kinoshita A, Onoda H, Fushiya N, Koike K, Nishino H, Tajiri H. Staging systems for hepatocellular carcinoma: current status and future perspectives. World J Hepatol 2015;7:406-24.

4. Yu SJ. A concise review of updated guidelines regarding the management of hepatocellular carcinoma around the world: 2010-2016. Clin Mol Hepatol 2016;22:7-17.

5. Jang JY, Lee JS, Kim HJ, Shim JJ, Kim JH, Kim BH, et al. The general rules for the study of primary liver cancer. J Liver Cancer 2017;17: 19-44.

6. Kim T, Kim BW, Wang HJ, Lee HY, Won JH, Kim J, et al. Quantitative assessment of the portal pressure for the liver surgery using serological tests. Ann Surg 2016;264:330-8.

7. Chun YH, Kim SU, Park JY, Kim DY, Han KH, Chon CY, et al. Prognostic value of the 7th edition of the AJCC staging system as a clinical staging system in patients with hepatocellular carcinoma. Eur J Cancer 2011;47:2568-75.

8. Minagawa M, Ikai I, Matsuyama Y, Yamaoka Y, Makuuchi M. Staging of hepatocellular carcinoma: assessment of the Japanese TNM and AJCC/UICC TNM systems in a cohort of 13,772 patients in Japan. Ann Surg 2007;245:909-22.

9. Liang KY, Zeger SL. Longitudinal data analysis using generalized linear models. Biometrika 1986;73:13-22.

10. Yamanaka N, Okamoto E, Kuwata K, Tanaka N. A multiple regression equation for prediction of posthepatectomy liver failure. Ann Surg 1984;200:658-63.

11. Makuuchi M, Kosuge T, Takayama T, Yamazaki S, Kakazu T, Miyagawa S, et al. Surgery for small liver cancers. Semin Surg Oncol 1993;9:298-304.

12. Guglielmi A, Ruzzenente A, Conci S, Valdegamberi A, Iacono C. How much remnant is enough in liver resection? Dig Surg 2012;29: 6-17.

13. Shimada M, Takenaka K, Kawahara N, Kajiyama K, Yamamoto K, Shirabe K, et al. Surgical treatment strategy for patients with stage IV hepatocellular carcinoma. Surgery 1996;119:517-22.

14. Yamamoto K, Takenaka K, Kawahara N, Shimada M, Shirabe K, Itasaka $\mathrm{H}$, et al. Indications for palliative reduction surgery in advanced hepatocellular carcinoma. The use of a remnant tumor index. Arch Surg 1997;132:120-3.

15. Chirica M, Scatton O, Massault PP, Aloia T, Randone B, Dousset B, et al. Treatment of stage IVA hepatocellular carcinoma: should we reappraise the role of surgery? Arch Surg 2008;143:538-43.

16. Kim BW, Park YK, Kim YB, Wang HJ, Kim MW. Salvage liver transplantation for recurrent hepatocellular carcinoma after liver resection: feasibility of the Milan criteria and operative risk. Transplant Proc 2008;40:3558-61.

17. Graf D, Vallböhmer D, Knoefel WT, Kröpil P, Antoch G, Sagir A, et al. Multimodal treatment of hepatocellular carcinoma. Eur J Intern Med 2014;25:430-7.

18. Maida M, Orlando E, Cammà C, Cabibbo G. Staging systems of 
hepatocellular carcinoma: a review of literature. World J Gastroenterol 2014;20:4141-50.

19. Fong ZV, Tanabe KK. The clinical management of hepatocellular carcinoma in the United States, Europe, and Asia: a comprehensive and evidence-based comparison and review. Cancer 2014; 120:2824-38.

20. Kee KM, Wang JH, Lin CY, Wang CC, Cheng YF, Lu SN. Validation of the 7th edition TNM staging system for hepatocellular carcinoma: an analysis of 8,828 patients in a single medical center. Dig Dis Sci 2013;58:2721-8.

21. Lei HJ, Chau GY, Lui WY, Tsay SH, King KL, Loong CC, et al. Prognostic value and clinical relevance of the 6th Edition 2002 American Joint Committee on Cancer staging system in patients with resectable hepatocellular carcinoma. J Am Coll Surg 2006;203: 426-35.

22. Vauthey JN, Lauwers GY, Esnaola NF, Do KA, Belghiti J, Mirza N, et al. Simplified staging for hepatocellular carcinoma. J Clin Oncol 2002;20:1527-36.

23. Huang J, Zhang Y, Peng Z, Gao H, Xu L, Jiao LR, et al. A modified TNM-7 staging system to better predict the survival in patients with hepatocellular carcinoma after hepatectomy. J Cancer Res Clin Oncol 2013;139:1709-19.

24. Chan AC, Fan ST, Poon RT, Cheung TT, Chok KS, Chan SC, et al. Evaluation of the seventh edition of the American Joint Committee on Cancer tumour-node-metastasis (TNM) staging system for patients undergoing curative resection of hepatocellular carcino- ma: implications for the development of a refined staging system. HPB (Oxford) 2013;15:439-48.

25. Kudo M, Chung H, Osaki Y. Prognostic staging system for hepatocellular carcinoma (CLIP score): its value and limitations, and a proposal for a new staging system, the Japan Integrated Staging Score (JIS score). J Gastroenterol 2003;38:207-15.

26. Kudo M, Chung H, Haji S, Osaki Y, Oka H, Seki T, et al. Validation of a new prognostic staging system for hepatocellular carcinoma: the JIS score compared with the CLIP score. Hepatology 2004;40: 1396-405.

27. Ikai I, Takayasu K, Omata M, Okita K, Nakanuma Y, Matsuyama Y, et al. A modified Japan Integrated Stage score for prognostic assessment in patients with hepatocellular carcinoma. J Gastroenterol 2006;41:884-92.

28. Kitai S, Kudo M, Minami Y, Ueshima K, Chung H, Hagiwara S, et al. A new prognostic staging system for hepatocellular carcinoma: value of the biomarker combined Japan integrated staging score. Intervirology 2008;51 Suppl 1:86-94.

29. Yen YH, Changchien CS, Wang JH, Kee KM, Hung CH, Hu TH, et al. A modified TNM-based Japan Integrated Score combined with AFP level may serve as a better staging system for early-stage predominant hepatocellular carcinoma patients. Dig Liver Dis 2009; 41:431-41.

30. Meinecke AK, Welsing P, Kafatos G, Burke D, Trelle S, Kubin M, et al. Series: pragmatic trials and real world evidence: Paper 8. Data collection and management. J Clin Epidemiol 2017;91:13-22. 